\title{
SPECTRORADIOMETRIC INVESTIGATION OF THE TRANSMISSION OF VARIOUS SUBSTANCES, II
}

\author{
By W. W. Coblentz
}

\section{ABSTRACT}

This paper gives transmission data in the spectrum extending from $0.6 \mu$ to $3 \mu$, using a mirror spectrometer, a quartz prism, and a vacuum thermopile. The substances examined are a group of mineral, animal, and vegetable oils (containing fatty acids), nitrocellulose, bakelite, and selenite. It is shown that the absorption spectra of the oils are so nearly identical that they can not be used for detecting the adulteration of one oil with another. The paper concludes with an examination of the accuracy of the author's previous work using a rock-salt prism. It is found that, using the recently determined refractive indices of rock salt, the corrections to the observations of 1903 to 1905 are of the order of 0.01 to $0.02 \mu$ and hence negligible.

\section{CONTENTS}

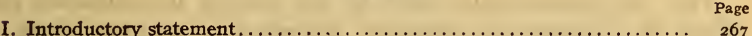

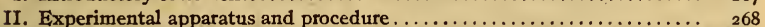

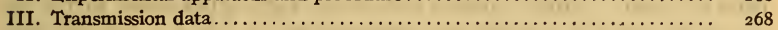

Appendix.-Concerning the accuracy of previous observations using a rock-salt

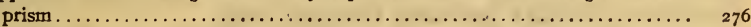

\section{INTRODUCTORY STATEMENT}

In previous papers data were published on the infra-red transmission of various substances particularly for wave lengths ${ }^{1}$ extending from 2 to ${ }^{5} \mu$. One of the important conclusions arrived at is that the great groups of chemical compounds-for example, alcohols, fatty acids, etc.-have characteristic absorption spectra. Hence one could hardly expect to detect spectroradiometrically the adulteration of a vegetable oil with a similar oil containing fatty acids; for example, cottonseed oil in olive oil. This conclusion was verified in a subsequent examination of pure olive oil, linseed oil, ${ }^{2}$ etc., in which the most conspicuous and characteristic absorption bands at 3 to $9 \mu$ occur so nearly at the same wave lengths that difficulties and uncertainties would arise in attempting to identify the substance producing them.

The object of the present paper is to record transmission data on the near infra-red spectrum, extending from 0.7 to $3 \mu$, thus supplementing previous work, and incidentally showing that this

1 Pub. No. 35, Carnegie Institution of Wash.; 1905. Also B. S. Bull., 2, p. 457; 1907.

2 B. S. Bull., 7, p. 648 ; 191 I. 
region of the spectrum is likewise unsuited for spectroradiometrically detecting adulteration of animal and vegetable oils of similar composition. Furthermore, from a consideration of the present and of the previously published data, it appears that, as a rule, superposed upon the bands of selective absorption there is a general or nonselective absorption (perhaps it is merely a widening of the spectral activity of the band of selective absorption) which increases rapidly with wave length. Consequently, in order to extend the observations beyond $2 \mu$, it is necessary to use thicknesses of material of the order of 0.1 to $0.01 \mathrm{~mm}$ to prevent complete opacity, whereas thicknesses of $\mathrm{I}$ to $\mathrm{Io} \mathrm{cm}$ are required in order to observe the absorption bands in the spectral region from 0.5 to $\mathbf{I} .5 \mu$. This appears to be true whether or not the substance-for example, dyestuffs-exhibits absorption in the visible spectrum.

\section{EXPERIMENTAL APPARATUS AND PROCEDURE}

The spectroradiometer used in the present investigation consisted of a mirror spectrometer described in previous work, ${ }^{3}$ a quartz prism ${ }^{4}$ which gives a relatively large dispersion in the region of 0.7 to $3 \mu$, and a vacuum thermopile. ${ }^{5}$ The source of radiation was a 500-watt, gas-filled tungsten lamp.

The absorption cell, $\mathbf{I} \mathrm{cm}$ in thickness, was provided with thin, highly polished quartz windows. For examining thin layers of material cells $0.3 \mathrm{~mm}$ or less in thickness were employed. They were made of plates of microscope cover glass between which was placed a U-shaped wire or tin foil, then glued.

The experimental procedure was to note the galvanometer deflection with and without the cell before the spectrometer slit. No correction was made for loss of energy by reflection and absorption in the glass and quartz windows, which loss is quite uniform to $2.4 \mu$. In some cases the transmission is higher than the theoretical value ( 92 per cent), from which it appears that there may have been internal reflection in the cell. However, since the main part of the problem was the accurate mapping of the location of the absorption bands, this is of no great importance.

\section{TRANSMISSION DATA}

The first observations to be described under this caption are on a series of animal and vegetable oils. These observations are illustrated in a series of spectral transmission curves, which are given

B. S. Bull, 10, p. 1; 1913. 'B. S. Sci. Papers, 16, p. $701 ; 1920$.

6. S. Sci. Papers, No. 413; ז9ax. 
at random, without special reference to the origin of the material (whether animal or vegetable) in order to show the great similarity of curves, and hence their unsuitability for detecting adulteration. All these oils, when in a I cm layer, exhibit three deep, wide bands in the region of I.I8, I.39, and I. $75 \mu$, respectively. Practically the only distinguishing characteristic is the absorption band at $0.65 \mu$ which occurs in vegetable oils containing chlorophyl. In the infra-red the writer found only slight indications of selective absorption, ${ }^{6}$ in the region of $0.73 \mu$, in an alcoholic solution of chlorophyl. Owing to the great opacity of alcohol at I.2 to I. $4 \mu$ it remains to be determined whether the observed increase in absorption of chlorophyl at I. $4 \mu$ is correct.

Olive Oil.-The transmission curve of a $1 \mathrm{~cm}$ layer of pure olive oil is given in curve $A$, Fig. I. Similar data were previously obtained $^{7}$ for the spectral region of 3 to Io $\mu$, the thickness of the layer being only $0.007 \mathrm{~mm}$. As already mentioned, the absorption band at $0.65 \mu$ is caused by chlorophyl.

Cottonseed Oil.-The sample examined was a standard commercial product (colorless and free from chlorophyl) known as Wesson oil. The transmission of a $\mathrm{I} \mathrm{cm}$ layer is given in curve $B$, Fig. I. From the close similarity of the transmission curve of cottonseed oil and that of olive oil it is evident that the spectroradiometric test can not be used to detect the adulteration of olive oil with cottonseed oil.

Peanut Oil.-The sample examined was a commercial product. The spectral transmission of a $\mathrm{I} \mathrm{cm}$ layer is given in curve $C$, Fig. I.

Tung Oil.-The sample examined was obtained from the chemistry division. It was made from nuts gathered especially for the purpose of procuring the pure product free from adulterants found in the commercial material, which is a liquid. The present sample was a white solid which liquefied on warming, after which it would remain liquid for several days.

The transmission of a $\mathbf{I ~ c m ~ l a y e r ~ o f ~ t h i s ~ m a t e r i a l , ~ i n ~ t h e ~ l i q u i d ~}$ state, is given in curve $A$, Fig. 2. Tung oil is adulterated with soya-bean oil, with cottonseed oil, and sometimes with fish oil. The transmission curves are so nearly identical that they can not be distinguished with certainty.

- Phy. Rev., 19, p. 27; 1904. In the transmission curves of a $20 \mathrm{~cm}$ layer of vegetable oils, published by Dr. K. S. Gibson. (The Cotton Oil Press, September, r920), the absorption band of chlorophyl at $0.66 \mu$ is very conspicuous. His curves of vegetable oils which are free from chlorophyl do not show the cbaracteristic band at 0.65 to $0.67 \mu$.

7 B. S. Bull., 7, p. 648 ; 19 rr. $55225^{\circ}-21-2$ 
Catfish Oil. - The transmission of a $\mathrm{I} \mathrm{cm}$ layer of catfish oil is given in curve $B$, Fig. 2 .

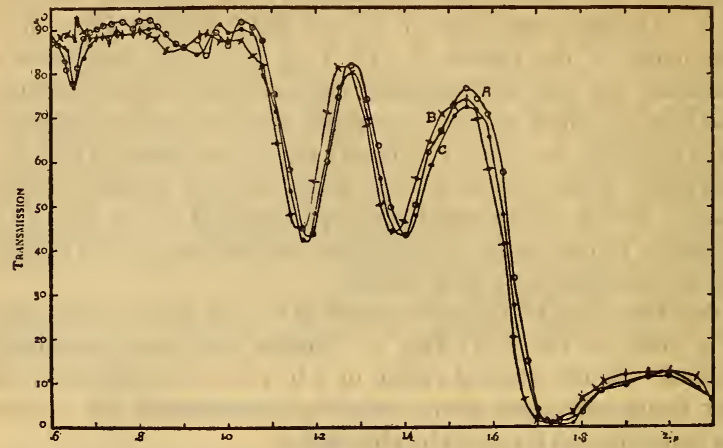

FIG. x.-A, olive oil; $B$, cotton seed oil; $C$, peanut oil

Soya-Bean Oil.-The special transmission of a $1 \mathrm{~cm}$ layer of commercial soya-bean oil is given in curve $C$, Fig. 2. Soya-bean oil is used to adulterate linseed oil.

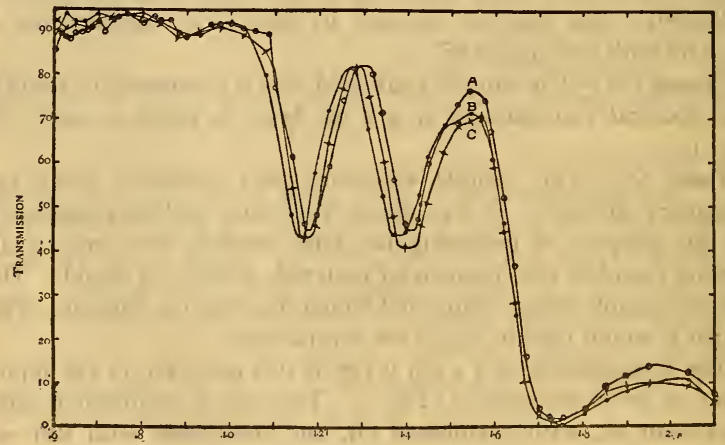

FIG. 2.- $A$, tung oil; $B$, catfish oil; $C$, soya bean oil

Lard Oil.-The spectral transmission of a I cm layer of clear lard oil is given in curve $A$, Fig. 3 . It is conspicuous for the sharpness of its absorption bands at $0.7,0.9,1.18$, and $1.41 \mu$. 
Fatty Acids of Linseed Oil.-This material was prepared in the chemistry division of this Bureau. It was brownish in color,

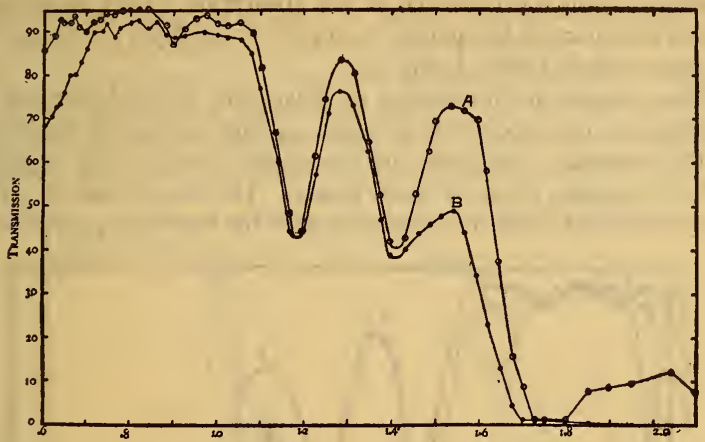

FIG. 3.-Lard oil, $A$; fatty acids of linseed oil, $B$

thus causing high absorption in the violet end of the visible spectrum. The spectral transmission of a $\mathrm{I} \mathrm{cm}$ layer is given in curve $B$, Fig. 3 .

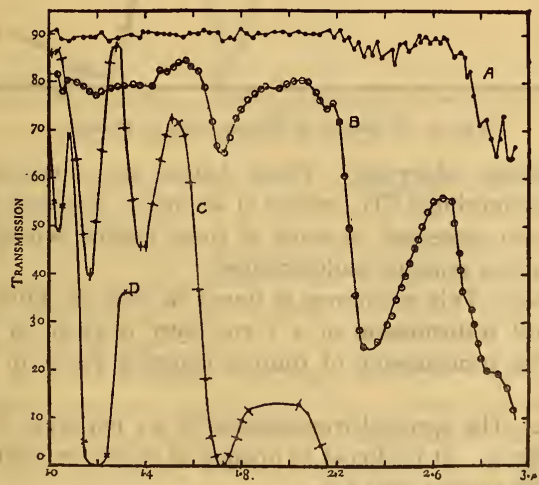

FIG. 4.-Linseed oil; thickness, $A=0.02 \mathrm{~mm}, B=0.37 \mathrm{~mm}, C=10 \mathrm{~mm}, D=100 \mathrm{~mm}$

Linseed Oil.-The spectral transmissions of various thicknesses of pure linseed oil are given in Fig. 4. Curve $A$ gives the transmission through a thickness of $0.02 \mathrm{~mm}$; curve $B=0.37 \mathrm{~mm}$; curve $C=10 \mathrm{~mm}$. 
Curve $D$ gives the transmission of a $10 \mathrm{~cm}$ layer of cottonseed oil. It is taken from a paper by Gibson ${ }^{8}$ to illustrate the effect of thickness upon the intensity of the absorption bands mentioned in the first part of the paper. Curve $C$ ( $\mathrm{I} \mathrm{cm}$ layer) is drawn to a larger scale in curve $A$, Fig. 5 .

These curves are interesting in illustrating a rapid increase in absorption in the spectrum beyond $\mathrm{I} .5 \mu$ with increase in thickness of the material. The wide unsymmetrical band at $2.3 \mu$ appears to be a complex group of small bands. The sharp band at $1.72 \mu$ is to be noticed, since it is harmonic with the bands at $3.43 \mu, 6.86 \mu$,

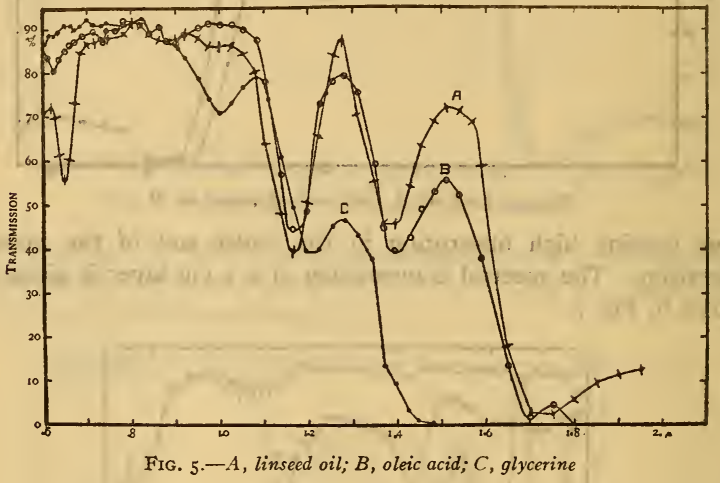

etc., previously observed. ${ }^{9}$ These bands are characteristic of substances containing $\mathrm{CH}_{2}$ groups of atoms. ${ }^{10}$ Whether the small band at $0.86 \mu$ observed in some of these curves belongs to this harmonic series remains undetermined.

Oleic Acid.-This substance is found in lard oil, olive oil, etc. The spectral transmission of a $\mathrm{I} \mathrm{cm}$ layer is given in curve $B$, Fig. 5. The transmission of thinner layers is given in previous papers. ${ }^{11}$

Glycerin.-The spectral transmission of a $\mathrm{I} \mathrm{cm}$ layer is given in curve $C$, Fig. 5. It is almost as opaque as water for radiations of wave lengths greater than $I .4 \mu$.

s Gibson, "The Cotton Oil Press," September, x920.

- B. S. Bulletin, 7, p. 649 ; rgrr.

10 Pub. No. 35, Carnegie Institution of Wash.; 1905.

11 Pub. No. 35, Carnegie Institution of Wash., p. $7 x$; 1905 . Also B. S. Bul., 7, p. 648; $191 x$. 
Nitrocellulose. - The samples examined were obtained under the trade names, Celluloid, Pyralin, and Viscoloid. ${ }^{12}$ They were thin, uncolored, and quite free from scratches and other imperfections. The thickness was closely the same for all samples, being $0.23 \mathrm{~mm}$ for celluloid and viscoloid, and $0.26 \mathrm{~mm}$ for pyralin.

The observed data are illustrated in Fig. 6, in which $A$ represents the observations on celluloid from 0.55 to $3 \mu$ and $A_{1}$ represents the same observations drawn to a larger scale in order to show the numerous fine absorption lines which occur in the region

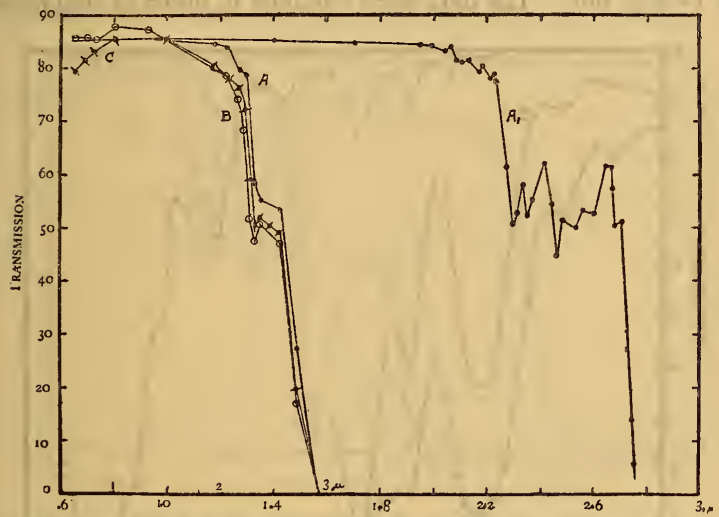

Frg. 6. $-A$ and $A_{1}$, celluloid; $B$, pyralin; $C$, viscoloid

of 2.3 to $2.6 \mu$. Curve $B$ gives the transmission of pyralin and curve $C$ that of viscoloid.

The data show that the transmission of all three kinds of the colorless material is high, being 80 to 85 per cent in the visible and in the infra-red to $3 \mu$. Beyond this point they are very opaque to infra-red rays.

Similar data on celluloid ${ }^{13}$ and on collodium ${ }^{14}$ were published by the writer some years ago when it was shown that these products of nitrocellulose have numerous absorption bands in the spectral region extending from 3 to $15 \mu$, which was the extent of the spectrum examined.

${ }^{12}$ Celluloid from The Celluloid Co., New York City; pyralin from E. I. du Pont de Nemours \& Co., Arlington, N. J.; and viscoloid from The Viscoloid Co., Leominster, Mass.

13 Pub. No. 97, p. 42, Carnegie Institution of Wash.; 1908.

14 Pub. No. 65, p. 60, Carnegie Institution of Wash.; 1906. 
In the ultra-violet these samples show considerable absorption at $0.365 \mu$ and are opaque to radiations of wave lengths less than $0.3 \mu$.

Judging from the behavior of other substances, the opacity of samples of celluloid, etc., which become discolored, is increased in the violet and ultra-violet, but not in the infra-red.

Selenite.-The spectral transmissions of various thicknesses of selenite, $\mathrm{CaSO}_{4}+2 \mathrm{H}_{2} \mathrm{O}$ are given in Fig. 7. For curve $A$, the thickness $t=0.1 \mathrm{I} \mathrm{mm}$; for curve $\mathrm{B}, t=2.80 \mathrm{~mm}$; and for curve $C, t=9.32 \mathrm{~mm}$. The data were obtained in order to supplement

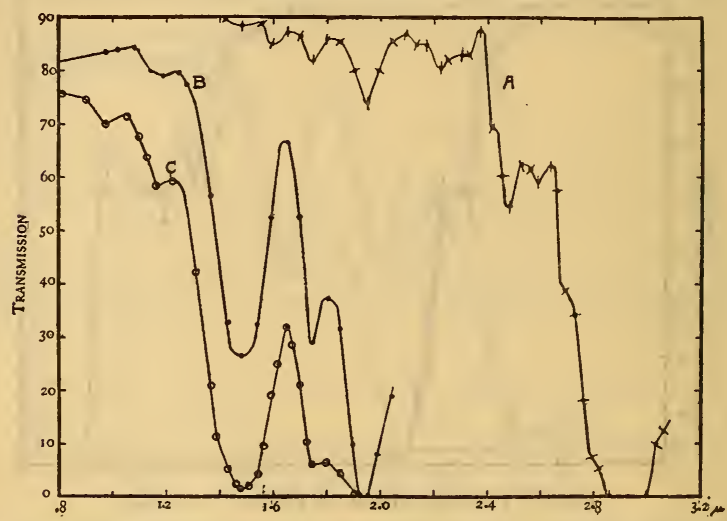

FIG. 7.-Selenite; thickness, $A=0.11 \mathrm{~mm}, B=2.80 \mathrm{~mm}, C=0.32 \mathrm{~mm}$

previous investigations of substances containing water of crystallization. ${ }^{15}$ In the present work the previously observed asymmetrical absorption band at 2.5 to $3 \mu$ is resolved into several distinct absorption bands. Considered in connection with the previous observations it appears that all these absorption bands are caused by water.

Paraffin Oil.-The material examined was a colorless liquid petrolatum (liquid paraffin) of American origin and great purity, used for medicinal purposes.

The second sample was a petroleum distillate (paraffin base) having a light brown color and a boiling point of 272 to $274^{\circ} \mathrm{C}$ at $2 \mathrm{~mm}$ pressure. 
The spectral transmissions of these two samples were found to be identical. The observations for $\mathrm{I} \mathrm{cm}$ layer are illustrated in curve $C$ of Fig. 8.

There are two sharp absorption bands, with maxima at $\mathrm{x} .2$ and I. $42 \mu$, respectively, which are so close to similar absorption bands in olive oil, etc. (Figs. I to 3 ), that it would be difficult to distinguish adulteration by spectroradiometric tests.

Benzol.-The spectral transmission of a $\mathrm{I} \mathrm{cm}$ layer of benzol, $\mathrm{C}_{6} \mathrm{H}_{6}$, is given in curve $A$, Fig. 8 . The data are of interest in connection with a previous examination ${ }^{16}$ of a thinner layer of

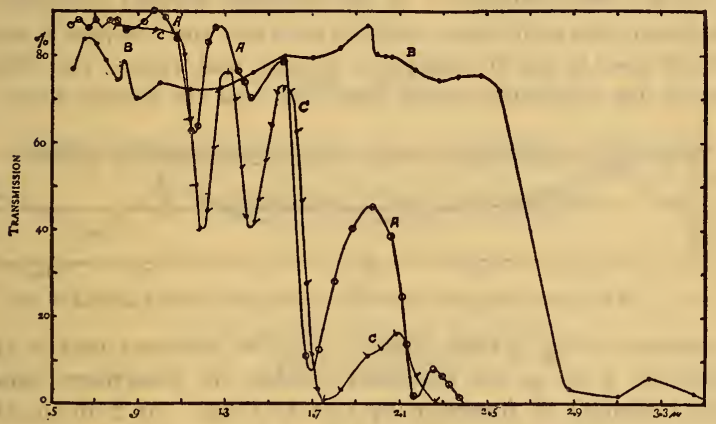

Fig. 8. $-A$, benzol; $B$, bakelite; $C$, mineral oil, paraffin base

this material which permitted an exploration of the bands at 2.18 and $2.49 \mu$, using a quartz prism. The $I \mathrm{~cm}$ layer is opaque to radiations of wave lengths greater than $2.5 \mu$. It is useful in locating absorption bands at $\mathrm{r} . \mathrm{I} 4$ and $\mathrm{r} .4 \mathrm{I} \mu$ (complex) which are barely perceptible in a thin layer of this material.

Bakelite.-This is a condensation product of formaldehyde and phenol. It was obtained as a varnish dissolved in acetone. A layer $0.04 \mathrm{~mm}$ in thickness was mounted upon a thin piece of glass and baked to remove the solvent. It has a high absorptivity in the ultra-violet but, as shown in curve $B$, Fig. 8 , shows no marked absorption bands (to $3 \mu$ ) in the infra-red, when used in a thin layer as just described. 
APPENDIX.-CONCERNING THE ACCURACY OF PREVIOUS OBSERVATIONS USING A ROCK-SALT PRISM

In 1903 to 1905 the writer determined the infra-red transmission of various substances using a rock-salt prism. At that time the dispersion of rock salt was not accurately known. In the region of $3 \mu$ there was considerable disagreement in the refractive indices as determined by various observers, and the writer used a smooth curve drawn through the various determinations. ${ }^{17}$

In the meantime a new determination ${ }^{18}$ was made of the refractive indices of rock salt, and recently a study was made of all the data available. ${ }^{10}$ It was therefore deemed desirable to recalculate the calibration curve of rock salt and compare it with the one used in the investigations of 1903 and 1905 . The difference in the calibration curve then used and the correct curve is

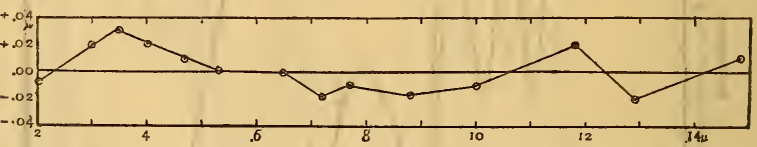

FIG. 9. -Wave length corrections to maxima of absorption bands published in 1905

illustrated in Fig. 9 from which it may be observed that in the region of 3 to $4 \mu$ the published maxima of absorption bands should perhaps be increased by 0.01 to $0.02 \mu$. At 7 to ro $\mu$ the published maxima should be decreased by o.or $\mu$. These corrections are so small that they appear to be negligible in comparison with the errors of observation resulting from changes in temperature of the prism and the small dispersion used.

That these data are free from large systematic errors is indicated by comparison with similar observations using a fluorite prism which has almost three times the dispersion of rock salt in the spectral region from 3 to $8 \mu$. For example, the maxima of characteristic absorption bands at 3.43 and $6.86 \mu$, first observed with the rock-salt prism, in substances containing $\mathrm{CH}_{2}$ and $\mathrm{CH}_{3}$ groups, are in good agreement with similar maxima subsequently observed with a fluorite prism.

Washington, April I, I92I.

${ }_{17}$ Pub. No. 35, Camegie Institution of Wash.; 1905 (Investigation of Infra-red Spectra, p. x32).

${ }^{18}$ Paschen, Ann. der Phys., 4, pp. 120 and 1029; 1908.

${ }^{29}$ B. S. Sci. Papers, 16, p. 701; 1920. 